\title{
Towards High-level Software Approaches to Reduce Virtualization Overhead for Parallel Applications
}

\author{
Stijn Schildermans and Kris Aerts \\ KU Leuven, Diepenbeek Campus \\ Department of Computer Science, ACRO - DTAI \\ Agoralaan 8, 3590 Diepenbeek, Belgium \\ Email: \{stijn.schildermans, kris.aerts\}@kuleuven.be
}

\begin{abstract}
Due to its numerous advantages, the IT industry is moving more and more towards the cloud for hosting applications. Reducing the virtualization overhead inherent to cloud computing has therefore been a topic of much research and innovation, resulting in a drastic reduction of this overhead for most workloads.

For specific tasks however, challenges remain. One example is parallel, CPU-intensive workloads. Studies concerning this specific workload exist, but focus on low-level properties of the virtualization process, which are often out of reach of the application programmer in commercial cloud environments. Therefore, we aim to approach virtualization overhead from an application architecture and implementation perspective, and provide guidelines for application programmers to develop their software in such a way that they avoid virtualization overhead without the need for access to the hypervisor or specific hardware.

As a first step towards this goal, this paper offers a proof of concept applied to the dedup benchmark from the PARSEC benchmark suite, which is notorious for its high virtualization overhead. We provide an alternative implementation of this benchmark, which suffers negligible virtualization overhead compared to the original, thus increasing performance by up to $45 \%$.
\end{abstract}

Index Terms -Parallelization, Virtualization, PARSEC

\section{INTRODUCTION}

Efficient virtualization of the x86-platform is a longstanding challenge, because of limitations of the architecture itself [1]. Several approaches have been taken to overcome these limitations [1], [2]. The standard approach today is hardware-assisted virtualization [3]. Although this technology is generally very effective, for specific tasks such as parallel, CPU-bound workloads, significant virtualization overhead is still incurred. To eliminate this problem, it makes sense to improve the hypervisor, or the hardware-support for virtualization. Both these approaches have indeed proven effective, but due to the shift towards cloud computing in industry, their impact has somewhat diminished, since many cloud providers do not offer fine-grained control over the hypervisor, and offer only a limited selection of hardware platforms to choose from.

Because of these reasons, it is in many cases favorable to reduce virtualization overhead purely from an application design and implementation perspective wherever possible. Therefore, we aim to provide a set of guidelines and best practices facilitating the development of parallel applications incurring minimal virtualization overhead, without depending on the hypervisor or hardware.

\section{A. Contributions}

The main contribution of this paper is to provide a proof of concept of the aforementioned goal. We demonstrate the link between application source code and virtualization overhead by analyzing a well-known parallel algorithm that incurs significant virtualization overhead, namely the dedup workload from the PARSEC benchmark suite. We provide an alternative implementation of said workload, eliminating almost all of the identified overhead and thus improving performance in a virtualized environment significantly.

\section{BACKGROUND \& RELATED WORK}

This paper assumes basic knowledge of hardware-assisted (x86-)virtualization and parallelization concepts. This section elaborates on the current state of the art with regard to virtualization overhead for parallel applications, and describes the dedup benchmark.

\section{A. Virtualization overhead for parallel workloads}

The majority of the literature concerning virtualization overhead for parallel applications focuses on high-performancecomputing (HPC), e.g. [4], [5], [6], [7]. However, these papers focus on overhead induced by communication between nodes in distributed settings. Our work on the other hand encompasses overhead at the virtual machine (VM) level, ignoring interaction between VMs. The nature of the overhead at these two levels is completely dissimilar.

Papers focusing on said VM level are rarer, but do exist. Perhaps the most well-studied phenomenon in this area is the lock-holder preemption problem [8], [9]. Although this issue is relevant to us, its cause and solution are mostly located at the OS and hypervisor level. Our study aims to avoid this kind of phenomena from a more general perspective, eliminating the cause of this problem rather than suppressing its symptoms.

One example of a more general study regarding parallelism at the VM level is [10], which shows that multithreaded workloads in paravirtualized VMs suffer degraded performance compared to native execution. In [11], a similar assessment to the one presented here is done, but the focus 
is on the effects of page locality in NUMA machines. To our knowledge, the study closest to our work is [12]. The authors quantify the performance impact of virtualization on the PARSEC benchmark suite.

All the studies mentioned above have in common that they offer almost exclusively OS-, hypervisor- or hardwarelevel solutions to their respective problems, and largely omit application-level approaches. One exception is [12], where the impact of the locking mechanism used by the application is studied. Our study takes this application-level approach a step further, and aims to mitigate all overhead purely from a software architecture and implementation perspective.

\section{B. The dedup benchmark}

Dedup is one of 12 workloads from the PARSEC benchmark suite, which is designed for testing the performance of multicore processors [13]. Of all 12 workloads in PARSEC, dedup is known to induce the most virtualization overhead by far [12].

The dedup workload itself is an implementation of the well-known data deduplication algorithm, used for data compression in e.g. (redundant) backups of large data sets [14]. The algorithm is implemented as a pipeline consisting of the following stages:

1) Read the input file from disk and coarsely divide it into chunks;

2) Refine each chunk into smaller chunks;

3) For each chunk, identify duplicates using a global hash table;

4) Compress all unique chunks;

5) Write the output to disk. Unique chunks are written in compressed form, and duplicate chunks are replaced by their hash value.

All pipeline stages run in parallel. Additionally, stages 2, 3 , and 4 are data-parallel, and thus for these stages multiple threads can be created. It is recommended that each of these stages are assigned at least as many threads as there are logical CPUs available on the system, so the scheduler can accurately balance CPU time between the different pipeline stages [13]. The benchmark is written in $\mathrm{C}$ and uses pthreads as its parallelization mechanism.

\section{METHOD}

To achieve the goal described in section I-A, we start with a stand-alone version of the dedup workload ${ }^{1}$. As input data we use a collection of pdf-files that was replicated several times, and then collected in a tar-archive. The total input size is 600 MB.

Our chosen hypervisor is KVM, running on Ubuntu 16.04. The test-system is a NUMA-server with 2 Skylake-era Intel Xeon CPUs, each with 8 physical and logical cores. The system has 2 banks of 16 GB of RAM.

In [12], perf $\mathrm{kvm}$ was used to profile the execution of the dedup benchmark. The authors noticed that almost all

\footnotetext{
${ }^{1}$ https://github.com/davidlohr/ezdedup
}

overhead is induced by a very large amount of inter-processor interrupts (IPIs). Therefore, this research starts with replicating their experiment to verify their findings.

We perform our experiments in two settings: with a single VM with 4 vCPUs, and with a single VM with 14 vCPUs. The number of threads used for each parallel stage of dedup has been set equal to the number of vCPUs in the system. We choose these numbers so we can see the effect of drastically increasing the core count and adding NUMA-properties to the execution of the algorithm. By using no more than 14 vCPUs, we minimize the chances of contention between the VM and background processes on the host.

A relatively recent technology implemented in modern Intel Xeon CPUs, namely APICv, drastically reduces the overhead induced by IPIs, as noted by [12]. Since at the time of writing APICv is still only supported by the latest generations of Xeon CPUs, we deem it relevant to perform our experiments with APICv enabled as well as disabled. After all, at the time of writing, some cloud providers still do not offer platforms that support APICv by default in all regions. A prominent example is Google 2 .

To achieve our research objective, it is paramount to link the IPIs mentioned above to the source code of dedup. Since sending IPIs requires ring 0-privileges, the source of the IPIs must be somewhere in the kernel. Therefore it makes sense to analyze the system calls made by dedup. We use strace for this purpose.

Once we have an idea of the overhead and system calls performed, we link them to each other and the dedup source code by analyzing the dedup, Linux kernel and glibc source code, as well as any relevant literature.

After the overhead has been identified, we must reimplement the dedup benchmark in such a way that we avoid inducing overhead as much as possible. We perform the experiments described above with our new implementation to verify the effectiveness of our approach.

\section{RESULTS \& DISCUSSION}

This section follows the same structure as section III. First we discuss the overhead of dedup, after which we identify our approach to eliminate it. We discuss our alternative implementation, and show the effects of it on the overhead and execution time of the benchmark.

\section{A. Identifying the overhead}

There are two steps to identyifying the overhead of the dedup benchmark. Firstly, we analyze the IPIs performed by the workload. Secondly, we identify the system calls performed, determine their root cause, and link them to the IPIs sent. We also gain insight in how these system calls and IPIs relate to the source code of dedup.

1) Perf Events: Table I shows the relevant results of the experiments described in section III. The results are displayed as the average number of occurances of each event over 10 invocations of the dedup benchmark.

\footnotetext{
${ }^{2}$ https://cloud.google.com/compute/docs/regions-zones/
} 
TABLE I: Overhead of the dedup benchmark.

\begin{tabular}{|c|c|c|c|c|}
\hline & \multicolumn{2}{|c|}{ APICv enabled } & \multicolumn{2}{c|}{ APICv disabled } \\
\hline Event & 4 vCPUs & $\mathbf{1 4}$ vCPUs & 4 vCPUs & $\mathbf{1 4}$ vCPUs \\
\hline VM_EXIT & $800 \mathrm{~K}$ & $2.4 \mathrm{M}$ & $1.3 \mathrm{M}$ & $3.3 \mathrm{M}$ \\
\hline MSR & $680 \mathrm{~K}$ & $2 \mathrm{M}$ & $660 \mathrm{~K}$ & $1.9 \mathrm{M}$ \\
\hline APIC_IPI & $500 \mathrm{~K}$ & $1.2 \mathrm{M}$ & $470 \mathrm{~K}$ & $1.1 \mathrm{M}$ \\
\hline
\end{tabular}

Table I shows a significant amount of IPIs for all tested versions, increasing with core count. As stated in section III, it is known that IPIs are a significant form of virtualization overhead [15], [16]. For each IPI, a write to the interrupt control register (ICR) MSR is required. This operation always results in a VM_EXIT. Without APICv, another VM_EXIT is required to deliver this interrupt. APICv eleminates this last form of VM_EXITs, among other optimizations. Interested readers are referred to [17] for more details on APICv and IPIs in Intel x86. The important takeaway here is that APICv only reduces IPI-related VM_EXITs by $30-50 \%$, and is thus not (yet) a perfect solution, meaning it is still preferable to avoid sending IPIs altogether.

For the 4 vCPU configuration, about $85 \%$ of IPIs have vector $0 x F C$, and about $15 \%$ have vector $0 x F D$. For the 14 vCPU version, these numbers are resp. $65 \%$ and $35 \%$. The Linux kernel 4.13 source code defines these vectors as resp. CALL_FUNCTION_VECTOR and RESCHEDULE_VECTOR. The CALL_FUNCTION IPI is of great interest to us since it is used to perform translation lookaside buffer (TLB) shootdowns. The TLB is a small per-core CPU-cache that stores page table entries (PTEs) [18]. This can significantly reduce memory address translation time since a TLB hit eliminates the need for a page walk. However, TLB coherence has to be managed by the OS. This means that whenever a PTE is updated, all TLBs containing this PTE must be updated, requiring the aforementioned CALL_FUNCTION IPI to be sent to all CPUs containing this PTE. The RESCHED$U L E \_V E C T O R$ on the other hand is critical to scheduling. The scheduler might decide to schedule a new task on a(n idle) CPU different from the one it is executing on. When this happens, a RESCHEDULE IPI is sent.

As table I shows, there is no linear relationship between the number of (v)CPUs and the number of IPIs sent. The number of RESCHEDULE IPIs grows excessively in relation to the amount of threads/CPUs. This is due to the fact that when there are more CPUs in the system, there is a higher chance that a remote CPU is more eligible for running a new task than the CPU executing the rescheduling algorithm; e.g. because it is idle. By contrast, the number of CALL_FUNCTION IPIs increases with core count, but less than linearly. This is likely caused by the fact that more available CPUs means that tasks and their accompanying TLB contents are spread over more CPUs as a result of repeated context switching and task migration. However, it is unlikely that the same task is scheduled on every CPU in the system. This results in the lessthan-linear rise in the need for TLB shootdowns, as observed in table I.
TABLE II: System calls performed by dedup.

\begin{tabular}{|c|c|}
\hline Call & Count \\
\hline write & $480 \mathrm{~K}$ \\
\hline madvise & $370 \mathrm{~K}$ \\
\hline futex & $130 \mathrm{~K}$ \\
\hline mprotect & $50 \mathrm{~K}$ \\
\hline
\end{tabular}

2) System calls: Table II shows the most important system calls performed by dedup when natively run with 4 threads on our test system, measured using strace. Other configurations were also tested, and yield similar results.

Table II shows that 4 different system calls are called very often. These are the prime suspects for causing the large amount of IPIs discussed in section IV-A1. These calls are analyzed in detail below. Other system calls are performed no more than 1000 times and include mmap, munmap, brk, etc. These calls also induce overhead, but are too few in number to be significant.

a) Write: The large number of calls to write is no surprise, since each chunk has to be written to the output file, as described in section II-B. This system call will not send IPIs, but does induce VM_EXITs because of the I/O involved.

b) Madvise: The many calls to madvise are all being performed from within a library function used to apply GZIP compression to the unique chunks, and always have the argument MADV_DONTNEED. The root cause of this turns out to lie in glibc. Madvise(MADV_DONTNEED) is called by the C-standard free function to trim the top of a heap arena when a threshold (128 kB by default) is reached. If a program often frees memory at the top of the heap, this might result in many calls to madvise. This is a moderately known issue, and the proposed fix is to disable heap trimming [19], [20]. This however can decrease memory efficiency of the application. Working around this problem from a design perspective is therefore a better approach.

The implementation of madvise in the Linux kernel does indeed send a CALL_FUNCTION IPI. The function to be called is flush_tlb_func_remote, which does exactly what its name implies. This is necessary, because it is legal for the application to access these pages again, but the kernel might need to restore their content [21].

c) Futex: This system call is performed whenever there is contention on a lock. A thread calls futex either to notify the kernel it is waiting for a lock to be available and block, or to wake blocking threads when the current thread releases the lock. Reducing the number of futex calls thus means reducing contention, which is always desirable.

Contrary to madvise, the implementation of futex in the Linux kernel does not perform any IPIs directly, but it does often call the scheduler, since threads need to block or be awoken. This results in the RESCHEDULE IPIs discussed in section IV-A1.

d) Mprotect: Finally, mprotect is used to change the permissions of a memory page. Like madvise, this call is used implicitly by glibc. When malloc is called from a user- 
created thread and there is no eligible memory block in the free list, a large block of memory is fetched from the OS using the mmap system call, with permissions set to PROT_NONE. Using mprotect to change the permissions from NONE to READ/WRITE, this memory is later made available for use.

Since mprotect changes permissions on memory pages, it makes sense that this system call requires a TLB flush. Again looking at the Linux kernel source code, we found that mprotect does indeed send IPIs similar to those madvise sends and is therefore another major cause of virtualization overhead.

\section{B. Eliminating the overhead}

In section IV-A2 we identified that the vast majority of overhead incurred by the dedup benchmark is related to memory management and thread synchronization. The latter is a direct result of the high-level design of the algorithm, but the former can also be seen as an indirect result thereof. Because of the pipeline all memory is accessed by many threads by design, and the scheduler determines the eventual memory footprint. This leaves restricted room for optimization.

Our alternative implementation therefore focuses on dataparallelism, and eliminates the pipeline altogether. This removes almost all need for thread synchronization, and allows for better control of the usage of memory. The trade-of is that we sacrifice some scalability. The source code is freely available $^{3}$. Below we provide a general overview of our approach.

All stages of the pipeline are viewed as functions that are applied to the input data in sequential order. We use an optimized linked list to keep track of all chunks between stages. The structure of the list is manipulated from the main thread only, and each worker thread gets a predetermined set of chunks to process. All queues between pipeline stages are removed. We use a single thread pool with as many threads as logical cores in the system. We can safely limit ourselves to this number, since we no longer require the scheduler to balance pipeline stages, as opposed to the original [13]. Because we maintain the order of the chunks, the entire reordering stage of the original implementation can be skipped and after compression, a bitstream is directly generated from a large batch of chunks at once to be written to the output. All of this almost completely eliminates calls to futex, and drastically reduces the calls to write.

Next, we turn our attention towards madvise. In the original implementation, input is read in large chunks of $128 \mathrm{MB}$. These chunks are refined into smaller chunks, which are in fact pointers to a certain index of this large input chunk. Only when all chunks related to an input chunk have been processed, the large input chunk is freed. This means that when later stages allocate memory, they must often get it from the top of the heap, rather than the free list, inducing the calls to madvise when this new memory gets freed, as discussed earlier. We fix this by allocating a new memory buffer for each chunk in the fragmentation stage, rather than using pointers. These much

\footnotetext{
${ }^{3}$ https://github.com/StijnSchildermans/dedup_without_overhead
}

smaller chunks can be freed more quickly, and their memory can be recycled. The downside of this is that we have to copy the entire input file. Other approaches are possible, but are omitted here for brevity.

The last system call to be reduced is mprotect. As explained in section IV-A2, we must reduce the number of memory allocations, as well as return memory as quickly as possible. One place where we saw opportunity for this is in the compression stage. In the original implementation, for each chunk a buffer for the compressed data has to be allocated. Our threading approach however allows us to assign memory for many chunks at once. This means a drastic reduction in calls to mprotect. This approach is less straightforward with the original pipeline model, because of the larger amount of threads meaning a lot more synchronization and memory overhead.

Our implementation is significantly less complex from a design perspective than the original, as well as more concise in terms of implementation. Moreover, there is ample room for further improvement. For example, we can start the refinement stage in parallel with fragmentation, at the cost of reordering the chunks afterwards. However, we do not deem it relevant for this proof-of-concept paper to squeeze out maximum performance. After all, our focus is virtualization overhead rather than bare performance.

\section{Results}

This section elaborates on the effects of the improvements described in section IV-B. System calls, virtualization overhead, and execution time are discussed separately.

1) System calls: First of all table III describes the system calls our new implementation performs, in identical test conditions compared to table II.

TABLE III: System calls performed by our re-implementation of dedup.

\begin{tabular}{|c|c|}
\hline Call & Count \\
\hline write & 200 \\
\hline madvise & 1500 \\
\hline futex & 100 \\
\hline mprotect & $10 \mathrm{~K}$ \\
\hline
\end{tabular}

As expected, table III shows that the calls to write, madvise, and futex have been almost completely eliminated. The number of calls to mprotect has been reduced by $80 \%$. This can be further improved by increasing the batch size of the chunks when assigning memory buffers in the compression stage, as described in section IV-B. This however decreases memory efficiency. We found a batch size of 1000 to be an optimal balance between memory efficiency and calls to mprotect.

2) Virtualization Overhead: Table IV shows the overhead induced by our alternative version of dedup in a virtualized environment. The method used is identical to that for table I.

Table IV shows a reduction in the number of VM_EXITs of $90-97 \%$. IPIs were reduced by $97 \%$. For 4 and 14 vCPUs respectively, $95 \%$ and $80 \%$ of IPIs are TLB shootdowns, 
TABLE IV: Overhead of our alternative implementation of dedup for 4 and 14 threads/(v)CPUs.

\begin{tabular}{|c|c|c|c|c|}
\hline & \multicolumn{2}{|c|}{ APICv enabled } & \multicolumn{2}{c|}{ APICv disabled } \\
\hline Event & 4 vCPUs & $\mathbf{1 4}$ vCPUs & 4 vCPUs & 14 vCPUs \\
\hline VM_EXIT & $70 \mathrm{~K}$ & $100 \mathrm{~K}$ & $80 \mathrm{~K}$ & $120 \mathrm{~K}$ \\
\hline MSR & $30 \mathrm{~K}$ & $50 \mathrm{~K}$ & $30 \mathrm{~K}$ & $50 \mathrm{~K}$ \\
\hline APIC_IPI & $15 \mathrm{~K}$ & $30 \mathrm{~K}$ & $14 \mathrm{~K}$ & $30 \mathrm{~K}$ \\
\hline
\end{tabular}

indicating that we almost completely eliminated the overhead regarding thread synchronization. As expected, our achieved improvements are even larger with APICv turned off, and scale with core count.

3) Execution time: Table $\mathrm{V}$ shows the execution time of our reimplementation of dedup, relative to the original.

While we improved the general behavior of the code by reducing the amount of system calls, table $\mathrm{V}$ shows that this does not always impact the execution time positively. When natively run with 4 threads/CPUs, a minor slowdown is observed. This is expected, since in a native, single-socket environment the overhead of system calls and their inherent IPIs is limited, while we sacrificed the pipeline in return. In a virtualized environment however, the impact of said system calls and IPIs is much greater, due to the additional overhead of virtualization, as discussed in section IV-A1. This is clearly reflected in the execution time. With 14 vCPUs, this effect is even greater, since delivering IPIs takes much longer if the receiving CPU is located on another socket. Due to this, even in a native environment a large improvement in execution time is observed for 14 CPUs. APICv turns out to have little impact on eventual execution time, despite eliminating many IPI-induced VM_EXITS. This stresses the importance of good application design rather than relying solely on platform optimizations.

\section{CONCLUSiOn \& Future WORK}

We have shown that thread synchronization and memory management can be significant causes of virtualization overhead for CPU-intensive, parallel workloads. An alternative application design can have a dramatic effect on virtualization overhead and performance, especially for multi-socket systems. We provided strong evidence of this claim, reducing overhead and runtime of the dedup benchmark by up to $97 \%$ and $45 \%$, respectively.

We deem the results of this study relevant and interesting by themselves, and believe they will prove useful to application developers. However, it is difficult to derive generalized guidelines based on this work alone. As future work we will perform a broader study, and eventually provide said guidelines for developing parallel applications suffering minimal virtualization overhead, without relying on specific hypervisor or hardware features.

\section{ACKNOWLEDGMENT}

This research is part of the main author's $\mathrm{PhD}$ project. We would like to thank the anonymous reviewers for their helpful and constructive feedback.
TABLE V: Runtime of our dedup relative to the original.

\begin{tabular}{|c|c|c|c|}
\hline (v)CPUs & Native & APICv enabled & APICv disabled \\
\hline 4 & $105 \%$ & $80 \%$ & $80 \%$ \\
\hline 14 & $70 \%$ & $60 \%$ & $55 \%$ \\
\hline
\end{tabular}

\section{REFERENCES}

[1] D. Marshall, "Understanding full virtualization, paravirtualization, and hardware assist," VMWare White Paper, p. 17, 2007.

[2] K. Adams and O. Agesen, "A comparison of software and hardware techniques for x86 virtualization," ACM SIGARCH Computer Architecture News, vol. 34, no. 5, pp. 2-13, 2006.

[3] J. Fisher-Ogden, "Hardware support for efficient virtualization," University of California, San Diego, Tech. Rep, 2006.

[4] W. Huang, J. Liu, B. Abali, and D. K. Panda, "A case for high performance computing with virtual machines," in Proceedings of the 20th annual international conference on Supercomputing. ACM, 2006, pp. $125-134$.

[5] A. J. Younge, R. Henschel, J. T. Brown, G. Von Laszewski, J. Qiu, and G. C. Fox, "Analysis of virtualization technologies for high performance computing environments," in Cloud Computing (CLOUD), 2011 IEEE International Conference on. IEEE, 2011, pp. 9-16.

[6] E. Walker, "Benchmarking amazon ec2 for hig-performance scientific computing," ; login:: the magazine of USENIX \& SAGE, vol. 33, no. 5, pp. 18-23, 2008.

[7] J. P. Walters, V. Chaudhary, M. Cha, S. Guercio Jr, and S. Gallo, "A comparison of virtualization technologies for hpc," in Advanced Information Networking and Applications, 2008. AINA 2008. 22nd International Conference on. IEEE, 2008, pp. 861-868.

[8] T. Friebel and S. Biemueller, "How to deal with lock holder preemption," Xen Summit North America, vol. 164, 2008.

[9] J. Ouyang and J. R. Lange, "Preemptable ticket spinlocks: improving consolidated performance in the cloud," in ACM SIGPLAN Notices, vol. 48, no. 7. ACM, 2013, pp. 191-200.

[10] L. Youseff, K. Seymour, H. You, D. Zagorodnov, J. Dongarra, and R. Wolski, "Paravirtualization effect on single-and multi-threaded memory-intensive linear algebra software," Cluster Computing, vol. 12, no. 2, pp. 101-122, 2009.

[11] K. Z. Ibrahim, S. Hofmeyr, and C. Iancu, "Characterizing the performance of parallel applications on multi-socket virtual machines," in Proceedings of the 2011 11th IEEE/ACM International Symposium on Cluster, Cloud and Grid Computing. IEEE Computer Society, 2011, pp. $1-12$.

[12] X. Ding and J. Shan, "Diagnosing virtualization overhead for multithreaded computation on multicore platforms," in Cloud Computing Technology and Science (CloudCom), 2015 IEEE 7th International Conference on. IEEE, 2015, pp. 226-233.

[13] C. Bienia and K. Li, Benchmarking modern multiprocessors. Princeton University Princeton, 2011.

[14] "Dell emc glossary." [Online]. Available: https://www.emc.com/ corporate/glossary/data-deduplication.htm

[15] J. Ouyang, J. R. Lange, and H. Zheng, "Shoot4u: Using vmm assists to optimize tlb operations on preempted vcpus," ACM SIGPLAN Notices, vol. 51 , no. 7, pp. 17-23, 2016.

[16] M. K. Kumar, S. Maass, S. Kashyap, J. Veselỳ, Z. Yan, T. Kim, A. Bhattacharjee, and T. Krishna, "Latr: Lazy translation coherence," in Proceedings of the Twenty-Third International Conference on Architectural Support for Programming Languages and Operating Systems. ACM, 2018, pp. 651-664.

[17] I. Corporation. [Online]. Available: https://www.intel.com/content/dam/ www/public/us/en/documents/manuals/64-ia-32-architectures-softwaredeveloper-system-programming-manual-325384.pdf

[18] H. Wong, "Tlb and pagewalk coherence in x86 processors," Aug 2015. [Online]. Available: http://blog.stuffedcow.net/2015/08/pagewalkcoherence/

[19] M. Gorman, "Sourceware." [Online]. Available: https://sourceware.org/git/gitweb.cgi?p=glibc.git; $\mathrm{h}=$ c26efef9798914e208329c0e8c3c73bb1135d9e3

[20] YinKe, "Red hat." [Online]. Available: https://bugzilla.redhat.com/ show \_bug.cgi?id=1551902

[21] Linux, "madvise(2) - linux manual page," Sep 2017. [Online]. Available: http://man7.org/linux/man-pages/man2/madvise.2.html 